\title{
Tackling asthma phenotypes in community studies
}

\section{Peter G Gibson}

Episodic wheezing with variable airflow obstruction (asthma) defines a syndrome that is well recognised and much studied. It is now clear that this syndrome is heterogeneous in its clinical features, treatment response, prognosis and pathophysiological mechanisms. Causation remains elusive, yet the differences in asthma prevalence across the world, ${ }^{1}$ the effect of migration on changing asthma prevalence ${ }^{2}$ and the rise in asthma prevalence in the past decades highlight very potent gene and environmental effects that modify the clinical expression of asthma.

Studying asthma heterogeneity is a useful way to disentangle these complex issues. ${ }^{4}$ The intense focus on allergic mechanisms has given us very precise understanding of the Th2-eosinophil mechanistic pathway, effective and specific treatment-for example, anti-immunoglobulin E (IgE) monoclonal antibody treatment-and shown that a primary prevention strategy based on this approach is not likely to be beneficial.

Current attention is focusing on patterns of granulocyte infiltration, specifically the presence or absence of eosinophils and neutrophils. ${ }^{5}$ There is clear heterogeneity in these responses among adults with asthma, where a non-eosinophilic pattern of response is associated with more severe persistent asthma, non-response to corticosteroid therapy and certain specific trigger factors such as tobacco smoking and occupational exposures. Furthermore, it is also possible to link the granulocyte response patterns to key immunological mechanisms, such as eosinophils with Th2 lymphocyte responses and neutrophils with dysfunctional innate immune responses. ${ }^{6}$ This approach integrates basic mechanisms with clinical asthma heterogeneity to yield endogenous asthma phenotypes, or "endotypes".

While the application of the granulocyte phenotypes has proven useful in many settings, its application to epidemiological studies has remained elusive. This is predominantly because of a lack of

Correspondence to: Dr Peter G Gibson, Department of Respiratory and Sleep Medicine, John Hunter Hospital, Locked Bag 1, HRMC NSW 2310 Newcastle, Australia; peter.gibson@hnehealth.nsw.gov.au accessible markers to allow classification of inflammatory phenotype in large numbers of subjects. Induced sputum and bronchial biopsy are the standard methods for detecting granulocyte phenotype in clinical research settings, but these techniques have yet to be successfully applied in the field. Some groups have tried it in a small way, ${ }^{2}$ but they do not seem to be rushing back!

Nadif and colleagues (pages 374-80) use data from the Epidemiological Study on the Genetics and Environment of Asthma (EGEA) to describe a novel yet simple approach of using blood counts to define granulocyte inflammatory patterns in asthma. ${ }^{9}$ The appeal of the approach comes from the ease of sample collection. Peripheral blood can be obtained from subjects of all ages, using familiar and standardised collection procedures (venepuncture). The approach is biologically plausible since the infiltrating granulocytes in the airway are bone marrow-derived cells which access the airway via the circulation. The mechanisms controlling bone marrow release of granulocytes are different from those controlling chemotaxis of cells into tissue, ${ }^{10}$ which means that the blood phenotype will be less precise than a tissue phenotype derived from an airway sample. Future work will need to assess the agreement between tissue and blood phenotype classifications; however, it is likely that the increased sample size available in an epidemiological study can be used to overcome any imprecision in blood phenotype classification. Similarly, it will also be necessary to control the many factors that influence circulating granulocyte counts such as time of day, recent exercise and exposures.

Earlier work has examined the relationship between blood and airway cells in asthma. Pizzichini et al ${ }^{11}$ compared the discriminatory potential of induced sputum, brochoalveolar lavage (BAL) and blood eosinophil counts in asthma and found that although the sensitivity and specificity of sputum eosinophils was highest (area under the curve (AUC) 0.9), blood eosinophils also had good discriminatory potential that although numerically less (AUC 0.72) was not statistically different from sputum eosinophils. Blood eosinophils have also been demonstrated to relate to clinical asthma markers in many other studies. Studies with circulating neutrophils need to be done, whereas blood lymphocytes and their subsets do not seem to relate usefully to airway lymphocytes. ${ }^{12}$

Interestingly, the relative proportions of each blood inflammatory phenotype were similar to those reported in sputum studies, where the frequency of each pattern is ordered as paucigranulocytic, eosinophilic, neutrophilic and mixed pattern. ${ }^{5}$ The study was sufficiently large to have a high number of subjects with a mixed pattern, which in previous work have been allocated to the neutrophilic category. This has identified that some of the clinical features previously associated with neutrophilic asthma are most pronounced in mixed granulocytic asthma-that is, smoking, lower $\mathrm{FEV}_{1}$ (forced expiratory volume in $1 \mathrm{~s}$ ) and male gender-whereas others such as less severe airway hyper-responsiveness (AHR) are seen as features of neutrophilic or paucigranulocytic asthma. The study also shows that suppression of eosinophils by corticosteroid treatment cannot be the sole explanation for non-eosinophilic asthma since the majority of subjects with a non-eosinophilic pattern had not received corticosteroid therapy in the last 12 months. Perhaps of equal importance is that these phenotypes were defined in a body compartment separate from the airway. This highlights the growing recognition of the association between systemic inflammation and airway disease, which is increasingly recognised in chronic obstructive pulmonary disease (COPD), and now rediscovered in asthma.

Those with an eosinophilic pattern were clearly the sickest, a feature found in other studies, and the association of eosinophilia with serum IgE and bronchial hyperreactivity (BHR) confirms the mechanistic link between Th2 mechanisms and eosinophils as the endotype hypothesis predicts. The addition of increased neutrophils to raised eosinophils (mixed pattern, EOS ${ }^{\text {hi }}$ NEU ${ }^{\text {hi }}$ ) seems to worsen the clinical asthma severity, but not the COPD-like symptoms. Bronchitic symptoms of chronic phlegm and dyspnoea were more frequent in the neutrophil group, a feature we have also observed in clinic populations and in a macrolide treatment study of non-eosinophilic asthma. ${ }^{13}$ These observations point to potentially different mechanisms in these subjects that may involve mucus hypersecretion, and have clinical implications since these dominant symptoms of cough and 
mucus hypersecretion in non-eosinophilic asthma are not regularly assessed in asthma control questionnaires. This may explain why successful macrolide therapy improves asthma-related quality of life yet does not impact on asthma control score.

Will this approach be useful? Probably yes. Studies in respiratory epidemiology and other disciplines have found important relationships between circulating white cell count and mortality. ${ }^{14}{ }^{15}$ Parenthetically, this cautions the need carefully to examine the specificity of the results, particularly in populations where other influences may alter blood cell numbers, for example ageing or unrelated exposures. Several other studies have related white cell count to respiratory outcomes such as atopy ${ }^{16}$ and, importantly, the effect of exposures. ${ }^{17} 18$ To date, the characterisation of the clinical phenotype of subjects in genetic studies has been limited, and this may partly explain the inconsistency between studies and the small size of the genetic effects that have been identified. I have long puzzled over how scientists can define a person's genotype to the level of a single nucleotide yet accept a phenotype characterisation in the same individual that is as (im)precise as the answer to the question "Do you wheeze?". The solution to this problem does not lie in bigger and better gene machines that are sold with the promise of "personalised medicine"; it lies in better characterisation of the phenotype that can then be linked to genetic characterisation.

The use of circulating granulocyte counts is a simple yet effective way to give scientists access to better phenotypic classification in large-scale studies. It is one of those observations that prompts responses like, "anyone can do that", or "I wish I'd thought of that!". To which the reply might be, "Yes. So now lets make use of it".

\section{Competing interests: None.}

Thorax 2009;64:369-370. doi:10.1136/thx.2008.109710

\section{REFERENCES}

1. Weinmayr G, Weiland SK, Björkstén B, et al. Atopic sensitization and the international variation of asthma symptom prevalence in children. Am J Respir Crit Care Med 2007:176:565-74.

2. Gibson PG, Henry RL, Shah S, et al. Migration to a western country increases asthma symptoms but not eosinophilic airway inflammation. Pediatr Pulmonol 2003;36:209-15

3. London SJ, Romieu I. Gene by environment interaction in asthma. Annu Rev Public Health 2008 Nov 3. [Epub ahead of print]

4. Wenzel SE. Asthma: defining of the persistent adult phenotypes. Lancet 2006;368:804-13.

5. Simpson JL, Scott R, Boyle MJ, et al. Inflammatory subtypes in asthma: assessment and identification using induced sputum. Respirology 2006;11:54-61.

6. Simpson JL, Grissell TV, Douwes J, et al. Innate immune activation in neutrophilic asthma and bronchiectasis. Thorax 2007:62:211-8.

7. Anderson GP. Endotyping asthma: new insights into key pathogenic mechanisms in a complex, heterogeneous disease. Lancet 2008;372:1107-19.
8. Lemière $\mathbf{C}$, Walker $C, O^{\prime}$ Shaughnessy $D$, et al Differential cell counts in sputum in respiratory epidemiology: a pilot study. Chest 2001;120:1107-13

9. Nadif R, Siroux V, Oryszczyn M-P, et al. Heterogeneity of asthma according to blood inflammatory patterns. Thorax 2009;64:374-80.

10. Rosenberg HF, Phipps S, Foster PS. Eosinophi trafficking in allergy and asthma. J Allergy Clin Immunol 2007;119:1303-10.

11. Pizzichini E, Pizzichini MM, Efthimiadis A, et al. Measuring airway inflammation in asthma: eosinophils and eosinophilic cationic protein in induced sputum compared with peripheral blood. J Allergy Clin Immunol 1997;99:539-44.

12. Pizzichini E, Pizzichini MM, Kidney JC, et al. Induced sputum, bronchoalveolar lavage and blood from mild asthmatics: inflammatory cells, lymphocyte subsets and soluble markers compared. Eur Respir $J$ 1998;11:828-34

13. Simpson JL, Powell H, Boyle MJ, et al. Clarithromycin targets neutrophilic airway inflammation in refractory asthma. Am J Respir Crit Care Med 2008;177:148-55.

14. James $\mathbf{A L}$, Knuiman MW, Divitini ML, et al Associations between white blood cell count, lung function, respiratory illness and mortality: the Busselton Health Study. Eur Respir J 1999;13:1115-9.

15. Leng SX, Xue QL, Huang $Y$, et al. Baseline total and specific differential white blood cell counts and 5 -year all-cause mortality in community-dwelling older women. Exp Gerontol 2005:40:982-7.

16. Leung TF, Kong AP, Chan $\mathrm{H}$, et al. Association between obesity and atopy in Chinese schoolchildren. Int Arch Allergy Immunol 2009;149:133-140.

17. Menzies D, Nair A, Williamson PA, et al. Respiratory symptoms, pulmonary function, and markers of inflammation among bar workers before and after a legislative ban on smoking in public places. JAMA 2006;296:1742-8.

18. Glynn A, Thuvander A, Aune M, et al. Immune cell counts and risks of respiratory infections among infants exposed pre- and post-natally to organochlorine compounds: a prospective study. Environ Health 2008;7:62

\section{The art of replication}

\section{Michael Kabesch}

Hot topics in science, like fashion, come and go. Asthma genetics is no exception to this rule. Asthma candidate genes come and very few stay for good, changing from "candidate" gene to "disease" gene. Only an exclusive few survive a thorough replication process.

The asthma candidate genes studied in the replication approach by Blakey and colleagues $^{1}$ (pages $381-7$ ) were originally discovered by positional cloning, a technique based on linkage studies using microsatellite markers and subsequent fine mapping of these linkage signal loci in families. Until recently, when genome-wide association studies (GWAS) entered the field, this was

Correspondence to: Professor Michael Kabesch, Hannover Medical School, Center for Pediatrics, Clinic for Pneumology and Neonatology, Carl-Neuberg Strasse 1, D-30625 Hannover, Germany; Kabesch.Michael@mh.hannover.de the method of choice to detect novel asthma candidate genes. In contrast to candidate gene studies, which are hypothesis driven and always only as good as the underlying hypothesis, positionally cloned genes had the aura of being the better asthma candidate genes, as they were detected by a systematic genomic approach not potentially biased by the researcher's own belief in a hypothesis. Seven years after the first positionally cloned asthma candidate genes were published in 2002/2003, positional cloning has lost its nimbus. It became clear that these genes are no better candidates than those detected by hypothesis-driven approaches. Bias is not excluded by positional cloning. What counts at the end of the day in complex disease studies is replication and verification.

Interestingly, the interpretation of replication has become a matter of debate itself. Some argue that larger populations may not necessarily be better for replication of genetic signals in complex diseases. Disease definitions may be broader in large studies as phenotype assessment may be limited in larger studies. Environmental conditions in the replication study may be very different from the population where the initial result was acquired. As gene-environment interactions are an important factor in complex diseases and as asthma could be a syndrome with different underlying mechanisms, this diversity may influence the prospect for replication.

Another important issue in replication is that different but related phenotypes are sometimes used synonymously to argue for positive replication. This approach has been called "loose replication". For example, instead of replicating the association of single nucleotide polymorphism (SNP) A in gene 1 with asthma, an association of SNP B in gene 1 with elevated immunoglobulin $\mathrm{E}$ ( $\operatorname{IgE}$ ) in the replication study is observed. As elevated IgE may be related to asthma, this is counted as a loose replication of the initial finding. However, this seems rather like 